\title{
Dosimetric Comparison between Bone and Target Matching Considering Interfractional Prostate Motion in Volumetric Modulated Arc Therapy
}

\author{
Ryuta Nakahara ${ }^{1 *}$, Kentaro Ishii ${ }^{1}$, Nobuhide Wakai ${ }^{2}$, Ryu Kawamorita ${ }^{1}$, Wataru Okada ${ }^{1}$, \\ Shun Kishimoto', Kazuki Kubo' ${ }^{1}$, Toshifumi Nakajima ${ }^{1}$, Masatoshi Hasegawa ${ }^{2}$ \\ ${ }^{1}$ Department of Radiation Oncology, Tane General Hospital, Osaka, Japan \\ ${ }^{2}$ Department of Radiation Oncology, Nara Medical University, Kashihara, Japan \\ Email: *nakahara@tane.or.jp
}

How to cite this paper: Nakahara, R., Ishii, K., Wakai, N., Kawamorita, R., Okada, W., Kishimoto, S., Kubo, K., Nakajima, T. and Hasegawa, M. (2018) Dosimetric Comparison between Bone and Target Matching Considering Interfractional Prostate Motion in Volumetric Modulated Arc Therapy. International Journal of Medical Physics, Clinical Engineering and Radiation Oncology, 7, 47-60.

https://doi.org/10.4236/ijmpcero.2018.71005

Received: December 7, 2017

Accepted: February 10, 2018

Published: February 13, 2018

Copyright $\odot 2018$ by authors and Scientific Research Publishing Inc. This work is licensed under the Creative Commons Attribution International License (CC BY 4.0).

http://creativecommons.org/licenses/by/4.0/

\section{Open Access}

\begin{abstract}
Adequate matching methods are critical for accurate volumetric-modulated arc therapy (VMAT). We investigated the dosimetric differences in the target and organs at risk (OARs) between bone matching and target matching in patients with prostate cancer treated with VMAT. The relationship between the dosimetric differences and interfractional motion of the prostate was also evaluated. Forty patients with prostate cancer classified as intermediate risk were enrolled in a study to assess the differences in dosimetry between two matching methods. These patients were treated with VMAT and prescribed dose was $78 \mathrm{~Gy}$. The dose distribution was calculated using cone-beam computed tomography (CBCT) for this study. We selected clinical target volume (CTV) as the target, and the rectum and bladder as the OARs. The Dmean, D98, D95, and D2 to the target and V10-V70 to the OARs were calculated as different dose from target matching value minus bone matching value. Multiple regression analysis was used to evaluate the effect of interfractional motion of the prostate on the differences in dose. The CTV D95 values differed by $-0.22 \pm 1.01$ Gy (mean \pm standard deviation). Rectum and bladder V70 values differed by $4.6 \% \pm 7.2 \%$ and $-2.6 \% \pm 7.2 \%$, respectively. There was a correlation between interfractional motion of the prostate and the dose differences to OARs $\left(R^{2}=0.73-0.94\right)$. The dose differences to OARs also varied depending on the direction of the prostate's motion. We found that bone matching resulted in an increased rectal dose and high risk of decreasing dose to the CTV.
\end{abstract}




\section{Keywords}

Radiotherapy, Image-Guided, Radiation Dosage, Prostate

\section{Introduction}

Intensity-modulated radiotherapy (IMRT) for prostate cancer can deliver a more adequate dose distribution to the target while sparing normal tissue compared with conventional irradiation methods. This allows a higher dose to reach the target [1] [2]. The technique requires very high accuracy to minimize the chance of complications [3]. Guidelines recommend the use of image guidance [4], and studies have reported the resulting improvements in the accuracy of delivery and decreased complication rates [5] [6] [7].

There are various image guidance options for prostate cancer, including ultrasound [8], portal imaging with fiducial markers [9], megavoltage (MV) computed tomography (CT) [10] and kilovoltage (kV) cone-beam CT (CBCT) [11]. Nakamura et al. [12] reported that, in Japan, $47 \%$ of sites used bone matching with image-guided radiotherapy (IGRT) for prostate cancer treatment planning and $40 \%$ used target matching, with the other sites using fiducial markers. Target and bone matching methods differ in that target matching includes correction for interfractional organ motion of the prostate while bone matching does not. However, motion blur due to long acquisition times and image artifacts is an issue in target matching using CBCT [13].

Comparison of bone and target matching has found that they result in different doses to the rectum and bladder [14] [15] [16] [17]. The distance and direction of interfractional motion of the prostate may vary with different immobilization and preparation methods, and can vary among institutions [18]. Rijkhorst et al. [17] reported that the dosimetric effect of different matching methods is dependent on interfractional motion of the prostate in the anteroposterior direction. Knowledge of this effect is important when choosing a matching method. However, the optimal way of choosing a matching method has not been sufficiently investigated.

In this study, we assessed interfractional motion of the prostate and the resulting dosimetric effects on the target and organs at risk (OARs) using the bone and target matching methods.

\section{Materials and Methods}

\subsection{Patients Selection}

Forty patients with localized prostate cancer treated with IMRT at our institute between April 2012 and May 2013 were enrolled in this study. The patients were classified as "intermediate risk" based on the National Comprehensive Cancer Network (NCCN) prognostic risk grouping (www.nccn.org) that inclusions were clinical stage $\mathrm{T} 2 \mathrm{~b}$ or $\mathrm{T} 2 \mathrm{c}$, Gleason score of 7 or prostate-specific antigen of 10 - 
20. This study was approved by the Ethics Committee of Tane General Hospital. All patients gave written consent to participate in the study.

\subsection{Preparations}

Each patient was instructed to have a full bladder and empty rectum during CT

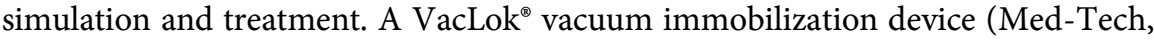
Orange City IA, USA) was used to fix each patient in the supine position. All patients were scanned on a 16-slice Optima CT580W ${ }^{\circledR}$ CT scanner (GE Medical Systems, Waukesha WI, USA) with a field of view of $50 \mathrm{~cm}$, tube voltage of 120 $\mathrm{kVp}$, tube current using the AutomA technique, matrix of $512 \times 512$ and slice thickness of $2.5 \mathrm{~mm}$.

\subsection{Image Guided}

Figure 1 shows the IGRT protocol used in this study. After setting up skin markers to position lasers for the replication of patient position, bone matching was performed using the ExacTrac ${ }^{\circledR}$ (BrainLAB, Heimstetten, Germany) X-ray $6 \mathrm{D}$ image-guided system (tube voltage $120 \mathrm{kV}$, tube current $160 \mathrm{~mA}$, time 160 $\mathrm{msec}$ ). We performed this in three planes and two axes corrections (anterior-posterior, superior-inferior, lateral, and pitching and rolling, respectively) using a $6 \mathrm{D}$ robotic couch. Subsequently, CBCT $(125 \mathrm{kV}$, tube current $80 \mathrm{~mA}$, time $13 \mathrm{msec}$, slice thickness $2.5 \mathrm{~mm}$, half fan mode) image data were acquired using the On Board Imager (OBI ${ }^{\oplus}$, Varian Medical Systems, Palo Alto CA, USA), which is a CBCT mounted on a Novalis $\mathrm{Tx}^{\otimes}$ linear accelerator (Varian), and target matching was performed manually by one medical physicist in three directions (anterior-posterior, superior-inferior, lateral). After bone matching by ExacTrac, the CBCT images, prior to undergoing target matching, were also used

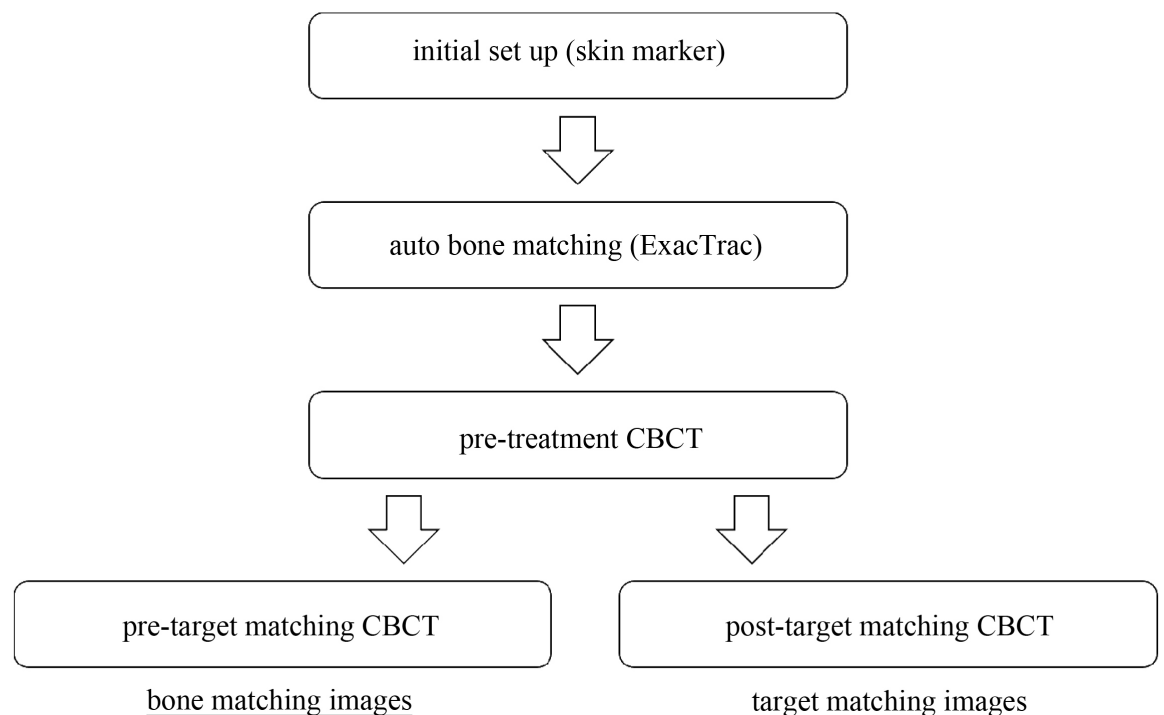

Figure 1. IGRT procedure in this study. After aligning skin markers with a laser, auto bone matching was performed using the ExacTrac system in all cases. Target matching was performed using $\mathrm{CBCT}$. $\mathrm{CBCT}=$ cone beam computed tomography. 
for bone matching, and the same images, after undergoing target matching, were defined as "target matching images." Image-guided volumetric-modulated arc therapy (VMAT) was planned using Eclipse ${ }^{\circledast}$ version 10.0 (Varian Medical Systems, Palo Alto CA, USA).

CBCT was acquired before every fraction. Five CBCT image datasets were randomly selected, and clinical target volume (CTV) and OARs were contoured on the selected images. The CTV was defined as the entire prostate plus the proximal $1.5 \mathrm{~cm}$ of the seminal vesicles. The planning target volume (PTV) was generated by adding an $8 \mathrm{~mm}$ margin to the CTV in all orientations except posteriorly, where a $5 \mathrm{~mm}$ margin was used. The prescribed dose was $78 \mathrm{~Gy}$ in 39 fractions. All plans were normalized to PTV mean dose. The rectum and bladder were contoured as solid organs. The rectum was segmented from the level of the ischial tuberosities to the rectosigmoid flexure, and the bladder was contoured from its apex to the dome. Contouring of the planning CT was performed by three radiation oncologists who were in charge of the patients. Contouring of CTV on CBCT was performed by one radiation oncologist and rectal and bladder contouring was performed by one medical physicist. The plan was calculated twice using the coordinates of bone matching and the results of target matching.

\subsection{Evaluations}

Interfractional motion of the prostate was defined as the difference in coordinates between bone matching and target matching. Dx is defined as the dose delivered to $\mathrm{x} \%$ of the volume and $\mathrm{Vx}$ is defined as the volume receiving $\mathrm{x}$ Gy of the dose. To investigate the dosimetric effect of the different matching methods, differences in Dx and Vx between target matching and bone matching were calculated. Changes in Dx and Vx were used as dosimetric indicators of the effect of the interfractional motion of the prostate on dose.

Dmean, D98, D95, D2 were used for CTV and V10, V20, V30, V40, V50, V60 and V70 were selected for OARs. Multiple linear regression analysis was used to evaluate the relationship between the difference in each of the dosimetric indicators and the interfractional motion of the prostate using SPSS Statistics ${ }^{\otimes}$ (IBM, Armonk NY, USA).

\section{Results}

The interfractional motion of the prostate in the anterior-posterior, superior-inferior and lateral directions measured (mean \pm standard deviation) $-1.9 \pm$ $2.8 \mathrm{~mm}, 0.3 \pm 2.2 \mathrm{~mm}$, and $-0.1 \pm 0.7 \mathrm{~mm}$ (Figure 2), where positive values indicate posterior, superior, and left directions, respectively. Figure 3 shows the differences in dose, where the target matching dose was subtracted from the bone matching dose. The bone matching dose produced higher increases in all dosimetric indicators to the rectum compared to the target matching dose, and resulted in lower doses to the CTV and bladder than the target matching dose. 
AP axis

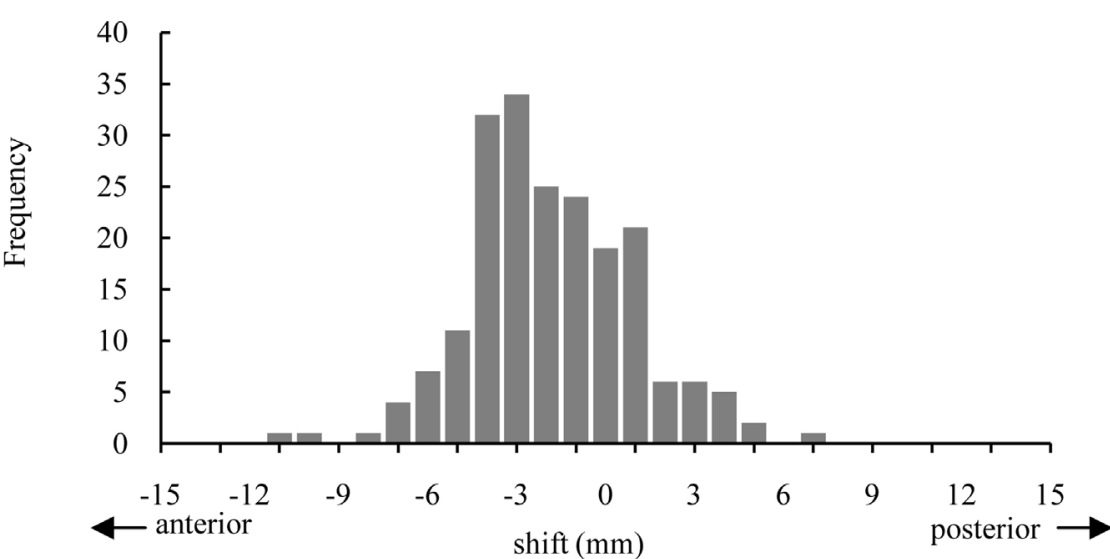

(a)

SI axis

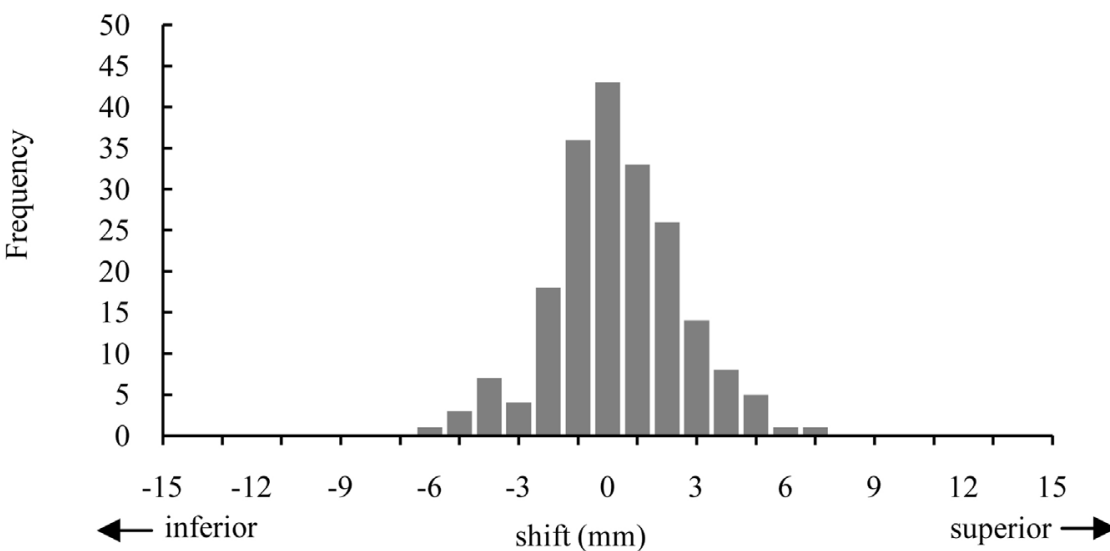

(b)

LR axis

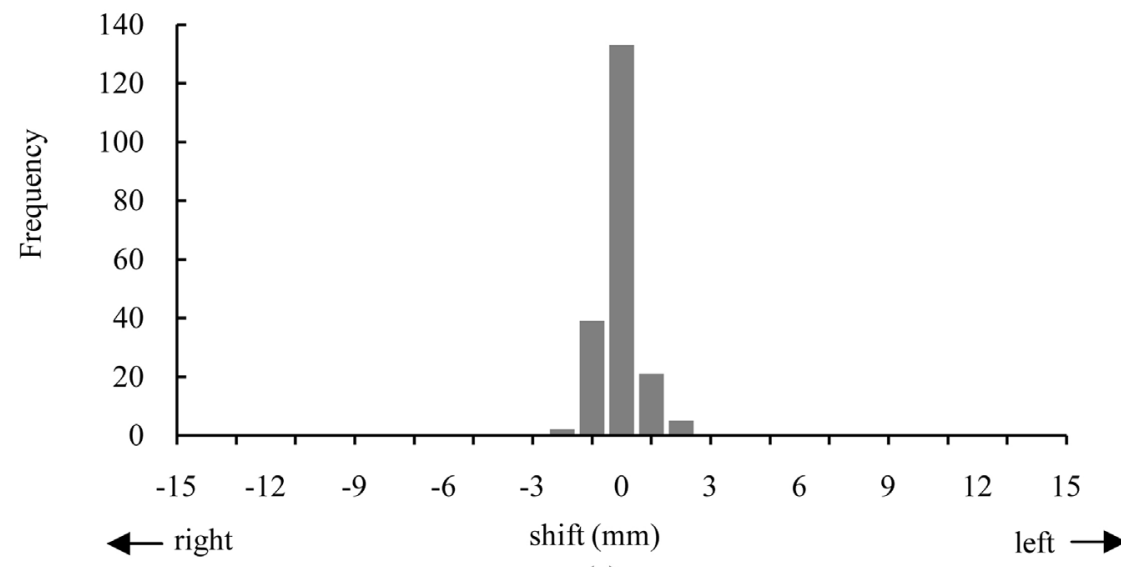

(c)

Figure 2. Histograms of interfractional motion of the prostate. (a) The anterior-posterior (AP) axis. Positive values indicate posterior. (b) The superior-inferior (SI) axis. Positive values indicate superior. (c) The left-right (LR) axis. Positive values indicate left.

Table 1 shows the results of multiple linear regression analysis of the relationship between interfractional motion of the prostate and the difference in 


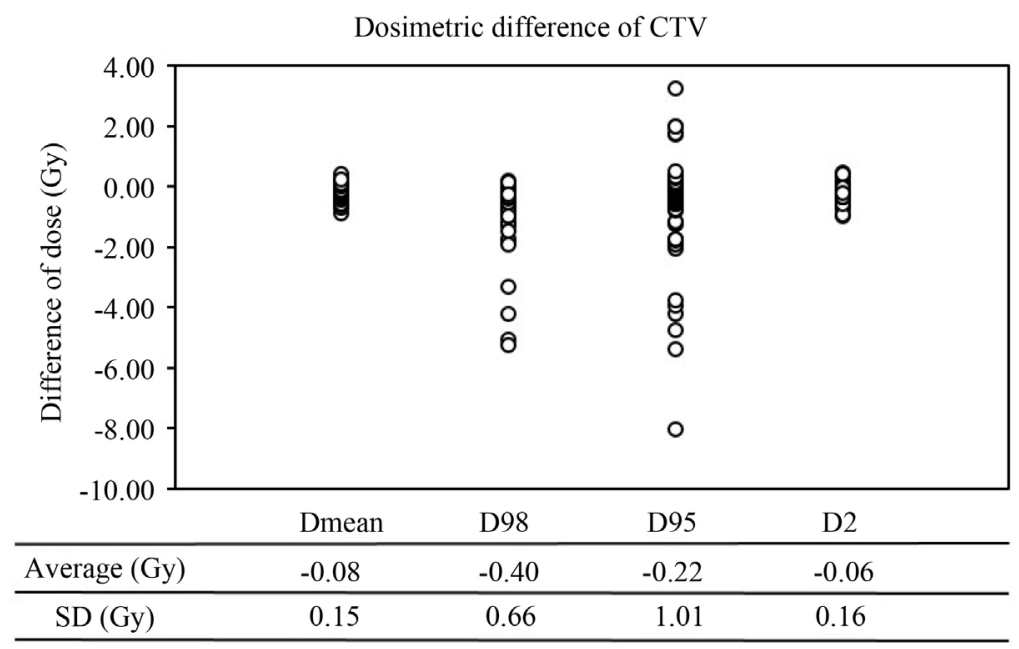

(a)

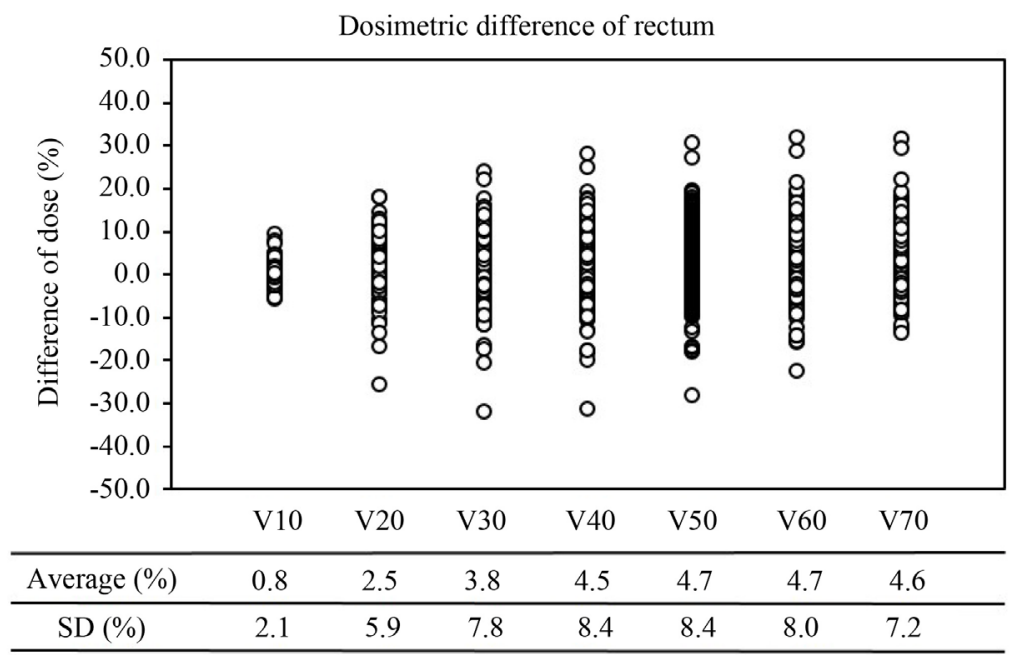

(b)

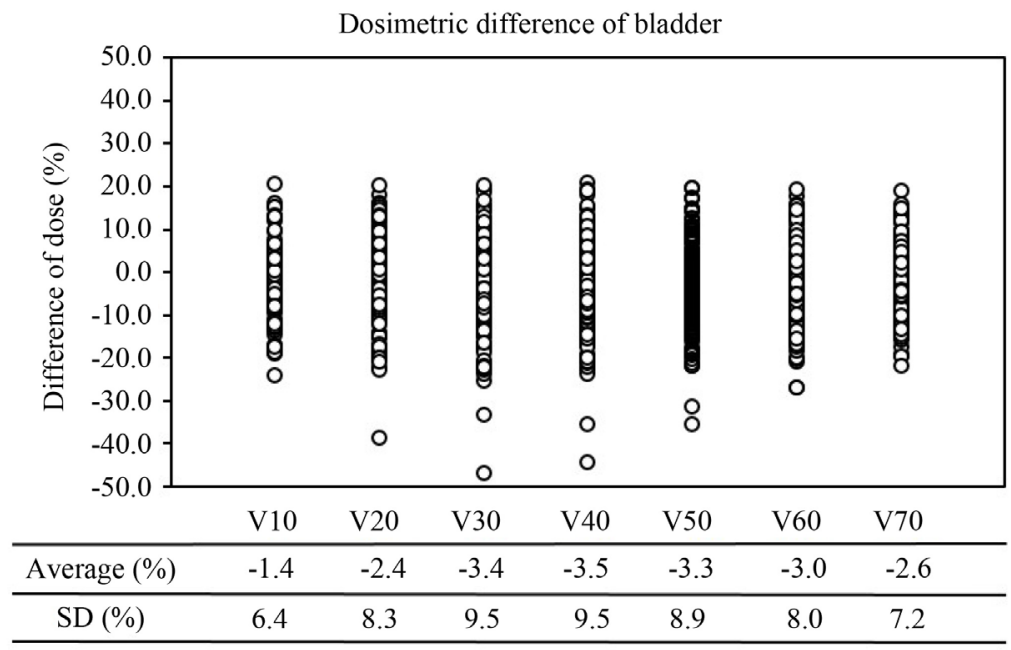

(c)

Figure 3. Dosimetric differences to the target and OARs by subtracting the target matching dose from the bone matching dose.(a) Dosimetric difference of CTV; (b) Dosimetric difference of rectum; (c) Dosimetric difference of bladder. 
Table 1. Multiple regression analysis of the relationship between interfractional motion of the prostate and the difference in dose to the rectum. Bold letters indicate the factor that affects the dose difference the most.

\begin{tabular}{|c|c|c|c|c|c|}
\hline & $\mathrm{R}^{2}$ & $\begin{array}{c}\text { Direction of } \\
\text { interfractional } \\
\text { motion of the prostate }\end{array}$ & $\begin{array}{l}\text { Regression } \\
\text { coefficient }\end{array}$ & $\mathrm{SD}$ & $\begin{array}{c}\mathrm{P} \\
\text { value }\end{array}$ \\
\hline \multirow{4}{*}{$\begin{array}{l}\text { Rectum } \\
\text { V10 }\end{array}$} & \multirow{4}{*}{0.38} & (constant) & -0.24 & 0.16 & 0.12 \\
\hline & & $\mathrm{AP}$ axis $(\mathrm{mm})$ & -0.63 & 0.07 & $<0.01$ \\
\hline & & SI axis $(\mathrm{mm})$ & -0.35 & 0.08 & 0.01 \\
\hline & & $\mathrm{LR}$ axis $(\mathrm{mm})$ & 0.09 & 0.18 & 0.61 \\
\hline \multirow{4}{*}{$\begin{array}{l}\text { Rectum } \\
\text { V20 }\end{array}$} & \multirow{4}{*}{0.73} & (constant) & -0.69 & 0.29 & 0.02 \\
\hline & & $\mathrm{AP}$ axis $(\mathrm{mm})$ & -1.70 & 0.12 & $<0.01$ \\
\hline & & SI axis $(\mathrm{mm})$ & 0.16 & 0.16 & 0.31 \\
\hline & & $\mathrm{LR}$ axis $(\mathrm{mm})$ & 0.50 & 0.33 & 0.13 \\
\hline \multirow{4}{*}{$\begin{array}{l}\text { Rectum } \\
\text { V30 }\end{array}$} & \multirow{4}{*}{0.87} & (constant) & -0.45 & 0.27 & 0.10 \\
\hline & & $\mathrm{AP}$ axis (mm) & -2.23 & 0.11 & $<0.01$ \\
\hline & & SI axis $(\mathrm{mm})$ & 0.61 & 0.15 & $<0.01$ \\
\hline & & LR axis (mm) & 0.41 & 0.31 & 0.19 \\
\hline \multirow{4}{*}{$\begin{array}{l}\text { Rectum } \\
\text { V40 }\end{array}$} & \multirow{4}{*}{0.92} & (constant) & -0.14 & 0.24 & 0.54 \\
\hline & & $\mathrm{AP}$ axis $(\mathrm{mm})$ & -2.38 & 0.10 & $<0.01$ \\
\hline & & SI axis $(\mathrm{mm})$ & 0.82 & 0.13 & $<0.01$ \\
\hline & & $\mathrm{LR}$ axis $(\mathrm{mm})$ & 0.41 & 0.27 & 0.13 \\
\hline \multirow{4}{*}{$\begin{array}{l}\text { Rectum } \\
\text { V50 }\end{array}$} & \multirow{4}{*}{0.93} & (constant) & 0.11 & 0.21 & 0.61 \\
\hline & & $\mathrm{AP}$ axis $(\mathrm{mm})$ & -2.33 & 0.09 & $<0.01$ \\
\hline & & SI axis $(\mathrm{mm})$ & 0.92 & 0.11 & $<0.01$ \\
\hline & & LR axis (mm) & 0.27 & 0.24 & 0.27 \\
\hline \multirow{4}{*}{$\begin{array}{l}\text { Rectum } \\
\text { V60 }\end{array}$} & \multirow{4}{*}{0.94} & (constant) & 0.41 & 0.19 & 0.03 \\
\hline & & $\mathrm{AP}$ axis $(\mathrm{mm})$ & -2.15 & 0.08 & $<0.01$ \\
\hline & & SI axis (mm) & 1.00 & 0.10 & $<0.01$ \\
\hline & & $\mathrm{LR}$ axis $(\mathrm{mm})$ & 0.12 & 0.22 & 0.58 \\
\hline \multirow{4}{*}{$\begin{array}{l}\text { Rectum } \\
\text { V70 }\end{array}$} & \multirow{4}{*}{0.93} & (constant) & 0.84 & 0.18 & $<0.01$ \\
\hline & & $\mathrm{AP}$ axis $(\mathrm{mm})$ & -1.83 & 0.08 & $<0.01$ \\
\hline & & SI axis (mm) & 1.01 & 0.10 & $<0.01$ \\
\hline & & LR axis (mm) & -0.17 & 0.21 & 0.42 \\
\hline
\end{tabular}

Vx denotes the difference between bone matching and target matching. $\mathrm{R}^{2}=$ coefficient of determination; $\mathrm{SD}=$ standard deviation; $\mathrm{AP}=$ anterior-posterior; $\mathrm{SI}=$ superior-inferior; $\mathrm{LR}=$ left-right.

dose to the rectum. The coefficient of determination indicates a correlation between interfractional motion of the prostate and the difference in each of the dosimetric indicators except V10 $\left(\mathrm{R}^{2}=0.73-0.94\right)$. The rectal dose was most influenced by anterior-posterior interfractional motion.

Table 2 shows the results of multiple linear regression analysis of the relationship between interfractional motion of the prostate and differences in 
Table 2. Multiple regression analysis of the relationship between interfractional motion of the prostate and the difference in dose to the bladder. Bold letters indicate the factor that affecs the dose difference the most.

\begin{tabular}{|c|c|c|c|c|c|}
\hline & $\mathrm{R}^{2}$ & $\begin{array}{c}\text { Direction of } \\
\text { interfractional } \\
\text { motion of the prostate }\end{array}$ & $\begin{array}{l}\text { Regression } \\
\text { coefficient }\end{array}$ & SD & $\begin{array}{c}\mathrm{P} \\
\text { value }\end{array}$ \\
\hline \multirow{4}{*}{$\begin{array}{l}\text { Bladder } \\
\text { V10 }\end{array}$} & \multirow{4}{*}{0.74} & (constant) & -0.40 & 0.31 & 0.20 \\
\hline & & $\mathrm{AP}$ axis (mm) & 0.13 & 0.13 & 0.32 \\
\hline & & SI axis (mm) & -2.39 & 0.16 & $<0.01$ \\
\hline & & LR axis (mm) & 0.15 & 0.35 & 0.66 \\
\hline \multirow{4}{*}{$\begin{array}{l}\text { Bladder } \\
\text { V20 }\end{array}$} & \multirow{4}{*}{0.86} & (constant) & -0.46 & 0.30 & 0.12 \\
\hline & & $\mathrm{AP}$ axis $(\mathrm{mm})$ & 0.55 & 0.12 & $<0.01$ \\
\hline & & SI axis (mm) & -2.94 & 0.16 & $<0.01$ \\
\hline & & $\mathrm{LR}$ axis $(\mathrm{mm})$ & 0.17 & 0.34 & $<0.62$ \\
\hline \multirow{4}{*}{$\begin{array}{l}\text { Bladder } \\
\text { V30 }\end{array}$} & \multirow{4}{*}{0.86} & (constant) & -0.35 & 0.34 & $<0.31$ \\
\hline & & $\mathrm{AP}$ axis $(\mathrm{mm})$ & 1.11 & 0.14 & $<0.01$ \\
\hline & & SI axis (mm) & -2.84 & 0.19 & $<0.01$ \\
\hline & & LR axis (mm) & 0.58 & 0.39 & 0.14 \\
\hline \multirow{4}{*}{$\begin{array}{l}\text { Bladder } \\
\text { V40 }\end{array}$} & \multirow{4}{*}{0.85} & (constant) & -0.13 & 0.35 & 0.71 \\
\hline & & $\mathrm{AP}$ axis $(\mathrm{mm})$ & 1.37 & 0.15 & $<0.01$ \\
\hline & & SI axis (mm) & -2.50 & 0.19 & $<0.01$ \\
\hline & & LR axis (mm) & 0.59 & 0.41 & 0.15 \\
\hline \multirow{4}{*}{$\begin{array}{l}\text { Bladder } \\
\text { V50 }\end{array}$} & \multirow{4}{*}{0.84} & (constant) & 0.01 & 0.34 & 0.99 \\
\hline & & $\mathrm{AP}$ axis $(\mathrm{mm})$ & 1.41 & 0.14 & $<0.01$ \\
\hline & & SI axis (mm) & -2.13 & 0.18 & $<0.01$ \\
\hline & & LR axis (mm) & 0.50 & 0.39 & $<0.21$ \\
\hline \multirow{4}{*}{$\begin{array}{l}\text { Bladder } \\
\text { V60 }\end{array}$} & \multirow{4}{*}{0.82} & (constant) & 0.09 & 0.32 & 0.77 \\
\hline & & $\mathrm{AP}$ axis $(\mathrm{mm})$ & 1.36 & 0.14 & $<0.01$ \\
\hline & & SI axis (mm) & -1.80 & 0.17 & $<0.01$ \\
\hline & & LR axis (mm) & 0.42 & 0.37 & 0.26 \\
\hline \multirow{4}{*}{$\begin{array}{l}\text { Bladder } \\
\text { V70 }\end{array}$} & \multirow{4}{*}{0.80} & (constant) & 0.27 & 0.31 & 0.39 \\
\hline & & $\mathrm{AP}$ axis $(\mathrm{mm})$ & 1.26 & 0.13 & $<0.01$ \\
\hline & & SI axis (mm) & -1.49 & 0.17 & $<0.01$ \\
\hline & & LR axis (mm) & 0.31 & 0.35 & 0.39 \\
\hline
\end{tabular}

$\mathrm{Vx}$ denotes the difference between bone matching and target matching. $\mathrm{R}^{2}=$ coefficient of determination; $\mathrm{SD}=$ standard deviation; $\mathrm{AP}=$ anterior-posterior; $\mathrm{SI}=$ superior-inferior; $\mathrm{LR}=$ left-right.

dose to the bladder. All differences in bladder dose correlated with interfractional motion of the prostate $\left(\mathrm{R}^{2}=0.74-0.86\right)$. The bladder dose was most influenced by superior-inferior interfractional motion.

Plots of the differences in CTV D95, rectum V70, and bladder V70 are shown in Figure 4, with the $\mathrm{x}$-axis indicating the direction of interfractional motion of the prostate with the strongest influence on the subsequent dose. The CTV D95 


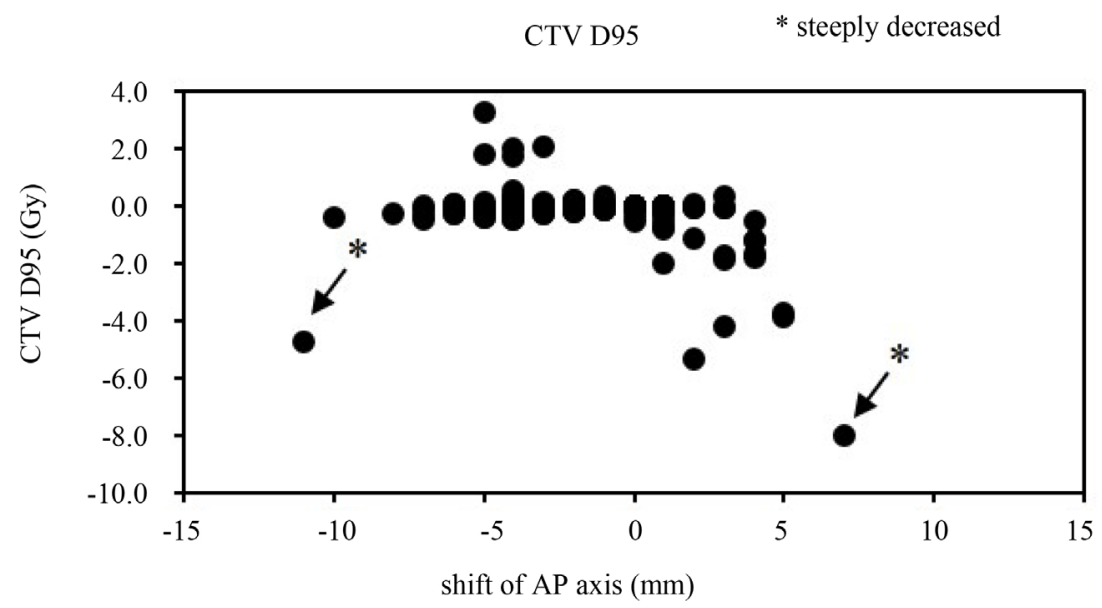

(a)

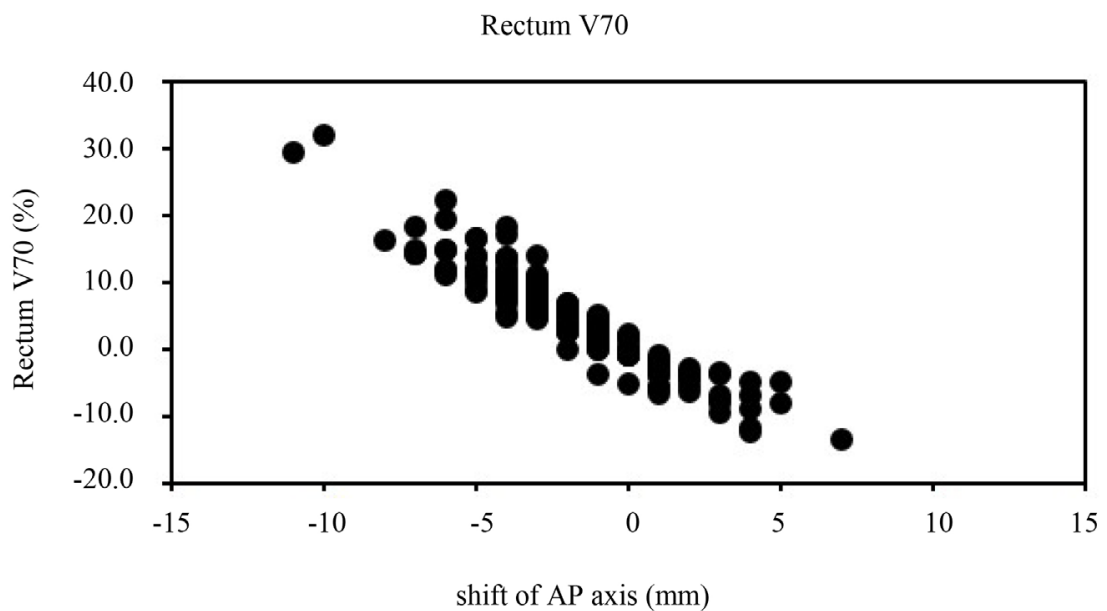

(b)

Bladder V70

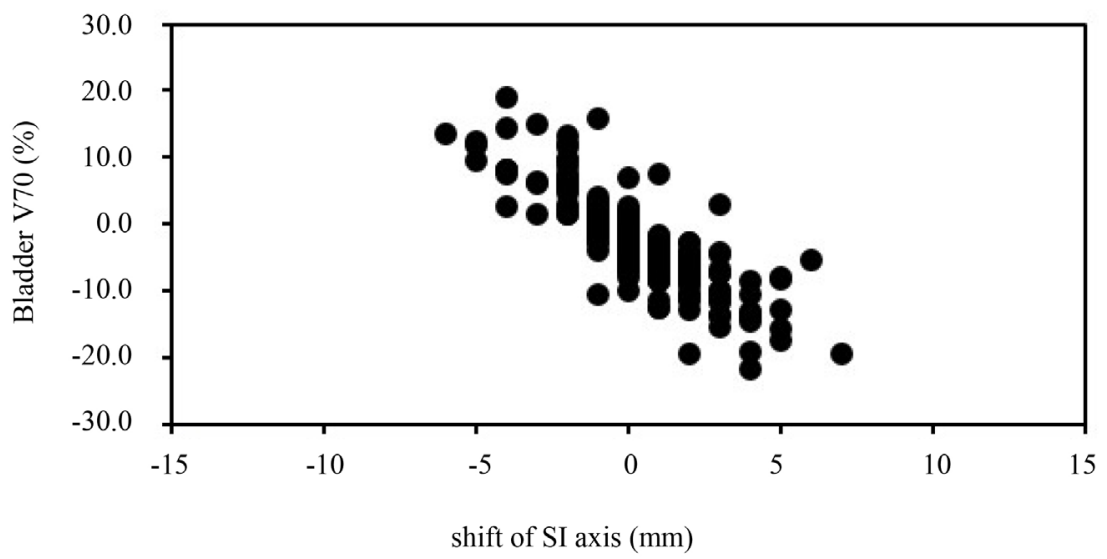

(c)

Figure 4. Representative dose changes to target and OARs. Positive values along the $\mathrm{x}$ axis indicate posterior ((a), (b)) and superior (c) directions. V70 and D95 denotes the difference between bone matching and target matching. The arrows represent the value of steeply dose decreasing of CTV when anterior-posterior interfractional motion of the prostate exceeded the PTV margin with bone matching was used. OARs = organs at risk. 
was steeply decreased when anterior-posterior interfractional motion of the prostate exceeded the PTV margin and bone matching was used. Motion in directions other than anterior-posterior did not exceed the PTV margin. Doses to the rectum and bladder were most affected by anterior and inferior interfractional motion of the prostate, respectively.

\section{Discussions}

Several studies have reported the effect of interfractional motion of the prostate on the target or OAR dose [18] [19] [20] [21]. Thongphiew et al. [14] investigated the use of CBCT in five fractions/week between bone and target matching and reported that bone matching resulted in a higher rectal dose than target matching. Using MVCT for comparison, Rivest et al. [15] reported that the rectal dose was higher with bone matching when interfractional motion of the prostate was in the anterior direction. Hirose et al. [16] compared rectal wall dose and bladder wall dose using СBCT and reported no differences between doses from bone matching and target matching. Our results suggest that the rectal dose is higher with bone matching due to anterior interfractional motion of the prostate. This corresponds with findings by Rivest et al. [15] and Rijkhorst et al. [17] showing that anterior interfractional motion of the prostate is associated with increased rectal dose.

We showed correlations between interfractional motion of the prostate and differences in rectal dose, excluding V10. Especially, it was suggest that in the case of where the interfractional motion of prostate moves $1 \mathrm{~mm}$ to posterior direction and use bone matching, each rectum dosimetric indicators excluded V10 increased about $2 \%(\mathrm{p}<0.01)$. Similarly, in the bladder, in the case of where the interfractional motion of prostate moves $1 \mathrm{~mm}$ to inferior direction and use bone matching, each bladder dosimetric indicators increased about $1 \%$ to $3 \%$ $(\mathrm{p}<0.01)$

Figure 5 shows both the rectal dose calculated at treatment planning in this study and the tolerance value of the rectal dose recommended by Quantitative Analyses of Normal Tissue Effects in the Clinic (QUANTEC) [22]. As the calculated dose at treatment planning increased, it approached the tolerance values of QUANTEC, with some V70 values exceeding the tolerance values. Due to the above reasons, it is important to consider this as a limiting factor when choosing matching methods.

The regression coefficients for the rectum and bladder were in opposite directions for the anterior-posterior and inferior-superior directions, indicating that the influence of the interfractional motion of the prostate on the difference in dosimetric indicators exerted opposite effects on the rectum and bladder. This relationship is due to the locations of these organs: the rectum is located posterior to the prostate while the bladder is located superior and anterior, such that when the prostate moves, the rectum and bladder move in the corresponding direction to occupy the space left by the movement of the prostate gland. 


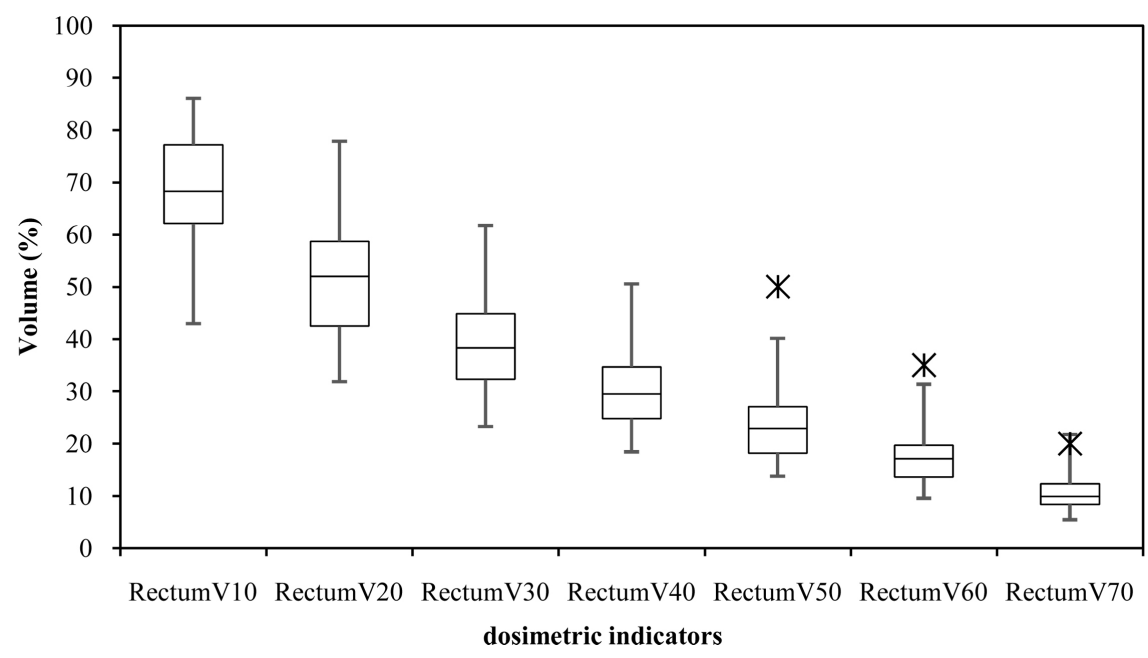

Figure 5. Relationship between rectal dose and tolerance value of QUANTEC. Box plots indicate treatment planning rectal doses and asterisks indicate the tolerance values of QUANTEC. Box plots with error bars indicate the median, minimum, 25th percentile, 75th percentile and maximum for each of the dosimetric indicators. QUANTEC $=$ Quantitative Analyses of Normal Tissue Effects in the Clinic.

In the CTV, there was only a small influence of interfractional motion of the prostate on the difference in dosimetric indicators. The lack of a clinically significant difference was presumably due to a sufficiently wide PTV margin setting. However, in some cases, posterior interfractional motion of the prostate caused a decrease in D95 to the CTV with bone matching, even with a distance shift within $5 \mathrm{~mm}$ of the posterior PTV margin (Figure 4). The above cases were planned to allow $<95 \%$ of the prescribed dose to the posterior aspect of the PTV; we observed an increase in D95 to CTV with bone matching due to anterior interfractional motion of the prostate when these posterior regions received $<95 \%$ of the prescribed dose.

Sato et al. [23] reported that target matching was superior to bone matching due to the ability to use a smaller PTV margin. Our results indicate that when bone matching is used with the same PTV margins, the rectal dose increases when the interfractional motion of the prostate is anterior, and the CTV is at higher risk of a decreased dose when the interfractional motion of the prostate is posterior. Figure 4 shows cases in which the interfractional motion of the prostate exceeded the PTV margin, with the CTV dose steeply decreasing with bone matching. Compared with target matching, bone matching has a lower exposure dose and better throughput. However for the above reasons, we recommend using target matching.

There are several limitations to this study. First, CBCT images were used in the dose calculation. Therefore, errors can occur in the dose calculation compared to using CT images for treatment planning. Onozato et al. [24] reported errors of $1.2 \%$ in the rectum and $1.0 \%$ in the bladder by comparing the precision of dose calculations in 10 prostate cancer patients. Furthermore, in this study, dose calculations were performed on the same $\mathrm{CBCT}$ images at different coor- 
dinates, and the differences were compared. Therefore, the effect of calculation errors should be sufficiently small compared to cases using treatment plan CT images. Additionally, the intrafractional motion of the prostate was not investigated; this is important when considering matching methodology.

\section{Conclusion}

Comparison of bone matching and target matching techniques showed that the CTV dose and OAR dose varied depending on the degree and direction of interfractional motion of the prostate. And using bone matching may lead increasing of rectum dose or decreasing of CTV dose. Thus, this suggests target matching is a better matching method than bone matching.

\section{Acknowledgements}

We thank Libby Cone, MD, MA, from DMC Corp. (www.dmed.co.jp) for editing the drafts of this manuscript.

\section{Funding}

This study was supported by Grant-in-Aid for Young Scientists (B) (No. 16K19236).

\section{References}

[1] Zelefsky, M.J., Fuks, Z., Happersett, L., Lee, H.J., Ling, C.C., Burman, C.M., Hunt, M., Wolfe, T., Venkatraman, E. and Jackson, A. (2000) Clinical Experience with Intensity Modulated Radiation Therapy (IMRT) in Prostate Cancer. Radiotherapy and Oncology, 55, 241-249. https://doi.org/10.1016/S0167-8140(99)00100-0

[2] Pollack, A., Hanlon, A., Horwitz, E.M., Feigenberg, S., Uzzo, R.G. and Price, R.A. (2003) Radiation Therapy Dose Escalation for Prostate Cancer: A Rationale for IMRT. World Journal of Urology, 21, 200-208. https://doi.org/10.1007/s00345-003-0356-x

[3] Chung, H.T., Xia, P., Chan, L.W., Park-Somers, E., and Roach, M. (2009) Does Image-Guided Radiotherapy Improve Toxicity Profile in Whole Pelvic-Treated HighRisk Prostate Cancer? Comparison between IG-IMRT and IMRT. International Journal of Radiation Oncology ${ }^{\star}$ Biology ${ }^{\star}$ Physics, 73, 53-60. https://doi.org/10.1016/j.ijrobp.2008.03.015

[4] Mohler, J.L. (2010) The 2010 NCCN Clinical Practice Guidelines in Oncology on Prostate Cancer. Harborside Press, LLC.

[5] Varadhan, R., Hui, S.K., Way, S. and Nisi, K. (2009) Assessing Prostate, Bladder and Rectal Doses during Image Guided Radiation Therapy-Need for Plan Adaptation? Journal of Applied Clinical Medical Physics, 10, 56-74. https://doi.org/10.1120/jacmp.v10i3.2883

[6] Zelefsky, M.J., Kollmeier, M., Cox, B., Fidaleo, A., Sperling, D., Pei, X., Carver, B., Coleman, J., Lovelock, M. and Hunt, M. (2012) Improved Clinical Outcomes with High-Dose Image Guided Radiotherapy Compared with Non-IGRT for the Treatment of Clinically Localized Prostate Cancer. International Journal of Radiation Oncology ${ }^{\star}$ Biology $^{\star}$ Physics, 84, 125-129.

https://doi.org/10.1016/j.ijrobp.2011.11.047 
[7] Valeriani, M., Bracci, S., Osti, M.F., Falco, T., Agolli, L., De Sanctis, V. and Enrici, R.M. (2013) Intermediate-Risk Prostate Cancer Patients Treated with Androgen Deprivation Therapy and a Hypofractionated Radiation Regimen with or without Image Guided Radiotherapy. Radiation Oncology, 8, 137. https://doi.org/10.1186/1748-717X-8-137

[8] Lattanzi, J., McNeeley, S., Hanlon, A., Schultheiss, T.E. and Hanks, G.E. (2000) Ultrasound-Based Stereotactic Guidance of Precision Conformal External Beam Radiation Therapy in Clinically Localized Prostate Cancer. Urology, 55, 73-78. https://doi.org/10.1016/S0090-4295(99)00389-1

[9] Barney, B.M., Lee, R.J., Handrahan, D., Welsh, K.T., Cook, J.T. and Sause, W.T. (2011) Image-Guided Radiotherapy (IGRT) for Prostate Cancer Comparing kV Imaging of Fiducial Markers with Cone Beam Computed Tomography (CBCT). International Journal of Radiation Oncology ${ }^{\star}$ Biology ${ }^{\star}$ Physics, 80, 301-305. https://doi.org/10.1016/j.ijrobp.2010.06.007

[10] Langen, K.M., Zhang, Y., Andrews, R.D., Hurley, M.E., Meeks, S.L., Poole, D.O., Willoughby, T.R. and Kupelian, P.A. (2005) Initial Experience with Megavoltage (MV) CT Guidance for Daily Prostate Alignments. International Journal of Radiation Oncology ${ }^{\star}$ Biology ${ }^{\star}$ Physics, 62, 1517-1524. https://doi.org/10.1016/j.ijrobp.2005.02.047

[11] Nijkamp, J., Pos, F.J., Nuver, T.T., De Jong, R., Remeijer, P., Sonke, J.-J. and Lebesque, J.V. (2008) Adaptive Radiotherapy for Prostate Cancer using Kilovoltage Cone-Beam Computed Tomography: First Clinical Results. International Journal of Radiation Oncology Biology Physics, 70, 75-82.

https://doi.org/10.1016/j.ijrobp.2007.05.046

[12] Nakamura, K., Akimoto, T., Mizowaki, T., Hatano, K., Kodaira, T., Nakamura, N., Kozuka, T., Shikama, N. and Kagami, Y. (2011) Patterns of Practice in Intensity-Modulated Radiation Therapy and Image-Guided Radiation Therapy for Prostate Cancer in Japan. Japanese Journal of Clinical Oncology, 42, 53-57. https://doi.org/10.1093/jjco/hyr175

[13] Bissonnette, J.P., Balter, P.A., Dong, L., Langen, K.M., Lovelock, D.M., Miften, M., Moseley, D.J., Pouliot, J., Sonke, J.J. and Yoo, S. (2012) Quality Assurance for Image-Guided Radiation Therapy Utilizing CT-Based Technologies: A Report of the AAPM TG-179. Medical Physics, 39, 1946-1963.

https://doi.org/10.1118/1.3690466

[14] Thongphiew, D., Wu, Q.J., Lee, W.R., Chankong, V., Yoo, S., McMahon, R. and Yin, F.F. (2009) Comparison of Online IGRT Techniques for Prostate IMRT Treatment: Adaptive vs. Repositioning Correction. Medical Physics, 36, 1651-1662. https://doi.org/10.1118/1.3095767

[15] Rivest, D., Riauka, T., Murtha, A. and Fallone, B. (2009) Dosimetric Implications of Two Registration Based Patient Positioning Methods in Prostate Image Guided Radiation Therapy (IGRT). Radiology and Oncology, 43, 203-212. https://doi.org/10.2478/v10019-009-0030-z

[16] Hirose, Y., Nakamura, M., Tomita, T., Kitsuda, K., Notogawa, T., Miki, K., Nakamura, K. and Ishigaki, T. (2014) Evaluation of Different Set-Up Error Corrections on Dose-Volume Metrics in Prostate IMRT using CBCT Images. Journal of Radiation Research, 55, 966-975. https://doi.org/10.1093/jrr/rru033

[17] Rijkhorst, E.-J., Lakeman, A., Nijkamp, J., de Bois, J., van Herk, M., Lebesque, J.V. and Sonke, J.-J. (2009) Strategies for Online Organ Motion Correction for Intensity-Modulated Radiotherapy of Prostate Cancer: Prostate, Rectum, and Bladder Dose Effects. International Journal of Radiation Oncology Biology Physics, 75, 1254- 
1260. https://doi.org/10.1016/j.ijrobp.2009.04.034

[18] Langen, K. and Jones, D. (2001) Organ Motion and Its Management. International Journal of Radiation Oncology Biology Physics, 50, 265-278. https://doi.org/10.1016/S0360-3016(01)01453-5

[19] Palombarini, M., Mengoli, S., Fantazzini, P., Cadioli, C., Degli Esposti, C. and Frezza, G.P. (2012) Analysis of Inter-Fraction Setup Errors and Organ Motion by Daily Kilovoltage Cone Beam Computed Tomography in Intensity Modulated Radiotherapy of Prostate Cancer. Radiation Oncology, 7, 56. https://doi.org/10.1186/1748-717X-7-56

[20] Nichol, A.M., Brock, K.K., Lockwood, G.A., Moseley, D.J., Rosewall, T., Warde, P.R., Catton, C.N. and Jaffray, D.A. (2007) A Magnetic Resonance Imaging Study of Prostate Deformation Relative to Implanted Gold Fiducial Markers. International Journal of Radiation Oncology Biology Physics, 67, 48-56.

https://doi.org/10.1016/j.ijrobp.2006.08.021

[21] Kim, J., Hammoud, R., Pradhan, D., Zhong, H., Jin, R.Y., Movsas, B. and Chetty, I.J. (2010) Prostate Localization on Daily Cone-Beam Computed Tomography Images: Accuracy Assessment of Similarity Metrics. International Journal of Radiation Oncology Biology Physics, 77, 1257-1265. https://doi.org/10.1016/j.ijrobp.2009.09.068

[22] Michalski, J.M., Gay, H., Jackson, A., Tucker, S.L. and Deasy, J.O. (2010) Radiation Dose-Volume Effects in Radiation-Induced Rectal Injury. International Journal of Radiation Oncology Biology Physics, 76, S123-S129. https://doi.org/10.1016/j.ijrobp.2009.03.078

[23] Sato, H., Abe, E., Utsunomiya, S., Kaidu, M., Yamana, N., Tanaka, K., Ohta, A., Obinata, M., Liu, J. and Kawaguchi, G. (2015) Superiority of a Soft Tissue-Based Setup using Cone-Beam Computed Tomography over a Bony Structure-Based Setup in Intensity-Modulated Radiotherapy for Prostate Cancer. Journal of Applied Clinical Medical Physics, 16, 239-245. https://doi.org/10.1120/jacmp.v16i5.5448

[24] Onozato, Y., Kadoya, N., Fujita, Y., Arai, K., Dobashi, S., Takeda, K., Kishi, K., Umezawa, R., Matsushita, H. and Jingu, K. (2014) Evaluation of On-Board kV Cone Beam Computed Tomography-Based Dose Calculation with Deformable Image Registration using Hounsfield Unit Modifications. International Journal of Radiation Oncology Biology Physics, 89, 416-423. https://doi.org/10.1016/j.ijrobp.2014.02.007 